\title{
La educación ambiental: una estrategia necesaria para la conservación de la geodiversidad del área serrana de Tandil, Argentina
}

\section{Environmental education: a necessary strategy for the conservation of geodiversity in the mountainous area of Tandil, Argentina}

\section{Aldo Ramos Schenck ${ }^{*}$}

DOI: https://doi.org/10.22458/rb.v32i1.3553

Recibido- Received: 19/02/2021 / Corregido- Revised: 08/03/2021 / Aceptado- Accepted: 15/03/2021

\section{RESUMEN}

La geodiversidad representa un gran valor. Es memoria de la Tierra, soporte de ecosistemas, recurso económico, científico y didáctico. Establece un vínculo con la cultura local, cohesionando y valorizando el territorio. Por esto, es necesario implementar medidas de conservación. Lamentablemente, la falta de conciencia y las presiones económicas terminan por afectar la geodiversidad. Por esta razón, es necesario elaborar estrategias de educación ambiental que posibilitan la geoconservación. El artículo presenta la problemática de la geodiversidad en una localidad de Argentina (Tandil) y la necesidad de otorgar un rol más preponderante a la educación ambiental para poder consolidar un proceso de conservación a largo plazo.

Palabras clave: geodiversidad; conservación; educación ambiental; concientización.

\section{ABSTRACT}

Geodiversity represents great value. It is Earth's memory, the support of ecosystems, and an economic, scientific, and didactic resource. It establishes a link with local culture by connecting and rating the territory. For this reason, conservation measures need to be implemented. Unfortunately, lack of awareness and economic pressures end up affecting geodiversity. For this reason, it is necessary to develop environmental education strategies that enable geo-conservation. The article presents the problem of geodiversity in Tandil, Argentina, and the need to give a more predominant role to environmental education in order to consolidate a long-term conservation process.

Key words: geodiversity; conservation; environmental education; awareness.

* Licenciado en Geografía. Máster en ecoaditorias. Posgrado en Educación Ambiental. Doctorando en Geografía (USAL). Universidad Nacional del Centro. Tandil. Argentina. aldogramos@gmail.com

ID: https://orcid.org/0000-0002-1113-4632 


\section{Introducción}

La geodiversidad, como explica Nieto (2001), constituye el sustrato sobre el que se desarrolla la vida y está conformada por estructuras sedimentarias, tectónicas, geomorfológicas, hidrogeológicas, entre otras, y de materiales geológicos. Lo anterior, significa que a través de ella podemos entender la historia de la Tierra. Pero esta importancia no siempre es reconocida por la sociedad, que no es consciente de las transformaciones generadas $y$ las repercusiones directas e indirectas de la falta de su conservación en aras de una economía no sostenible.

Frente a esta situación, se considera la necesidad de afrontar acciones a largo plazo que permitan sostener políticas de conservación de la geodiversidad. En este caso particular, se plantea la situación de la ciudad de Tandil (Argentina), rodeada hacia el sur y suroeste por serranías, objeto de diferentes usos (urbanos, recreativos, extractivos) que la ponen en riesgo.

En el trabajo se expresa la necesidad de abordar diferentes acciones en el marco de la educación ambiental en el nivel educativo formal, no formal e informal, desarrollando con mayor detalle una propuesta para aplicar en el nivel secundario, lo cual, a mediano y largo plazo, produce necesariamente un cambio en las actitudes y comportamientos de la sociedad, dejando así de considerar a las sierras como un objeto para comprender la geodiversidad como un componente fundamental para el desarrollo de la vida, de manera holística.

\section{Geodiversidad, conservación y educación ambiental}

"El ser humano es lo que la educación hace de él." Inmanuel Kant

El concepto de geodiversidad surge a finales de la década de 1990 aplicado a la gestión de espacios protegidos, evidenciando así la necesidad de un término que definiera y abarcara también los elementos abióticos del medio natural (Serrano Cañadas y Ruiz-Flaño, 2007).

La geodiversidad ha sido definida por Villalobos (en Volth, 2008, p. 2) como "la variabilidad que es posible encontrar en un determinado territorio en cuanto al número y la calidad de registros geológicos de interés para la ciencia y la educación". Johansson la define como la variación de fenómenos y procesos geológicos en un área definida y Stanley (en Nieto, L. M., 2001, p. 6) la considera como la "variedad de ambientes, fenómenos y procesos geológicos" que son "la infraestructura para la vida sobre la tierra". Las anteriores son algunas de las definiciones que de alguna forma están limitando el concepto de geodiversidad a la geología.

No obstante, por otro lado, algunos autores presentan una revisión del concepto de geodiversidad tal como Nieto (2001), quien la define de la siguiente forma.

[...] el número y la variedad de estructuras (sedimentarias, tectónicas, geomorfológicas, hidrogeológicas y petrológicas) y de materiales geológicos (minerales, rocas, fósiles y suelos), que constituyen el sustrato físico natural de una región, sobre las que se asienta la actividad orgánica, incluyendo la antrópica. (p. 7)

Desde esta misma perspectiva, se ha planteado que la geodiversidad es: 
[...] la variabilidad de la naturaleza abiótica, incluidos los elementos litológicos, tectónicos, geomorfológicos, edáficos, hidrológicos, topográficos y los procesos físicos sobre la superficie terrestre y los mares y océanos, junto a sistemas generados por procesos naturales, endógenos y exógenos y antrópicos, que comprende la diversidad de partículas, elementos y lugares. (Serrano Cañadas y RuizFlaño 2007, p. 82).

Según los autores mencionados, puede considerarse que los elementos que componen la geodiversidad sobre la superficie terrestre son: topografía,geología,geomorfología,hidrología ysuelos. La figura 1 presenta una síntesis de los elementos que integran la geodiversidad.

Por lo expuesto anteriormente, es necesario implementar estrategias de conservación, de la misma forma que se ha trabajado desde hace décadas con la biodiversidad. Lamentablemente, en muchas ocasiones las políticas de conservación de la geodiversidad no alcanzan para evitar las profundas transformaciones producidas por el ser humano. En este sentido, la educación ambiental tiene un rol muy importante. Es necesario que la sociedad en su conjunto entienda el valor de la geodiversidad, el cual, según TheodossiouDrandaki (2000, p. 119).

[...] sólo puede ser alcanzado a través de la conciencia colectiva, especialmente de la gente joven, que conociendo su patrimonio geológico local, llegará a apreciar su valor y, consecuentemente, a protegerlo. Es necesario pues educar a la gente en los ideales de la geoconservación y el patrimonio geológico.

Es primordial que la educación ambiental sea incorporada seriamente en los planes y programas de conservación. Como explica Theodossiou-Drandaki (2000, p. 119):

La educación es una herramienta sólida y por tanto una prioridad para conseguir la conservación de este tipo de patrimonio. Dicha educación debería abarcar desde los niveles de primaria y secundaria, hasta la universidad

Figura 1

Elementos integradores de la geodiversidad

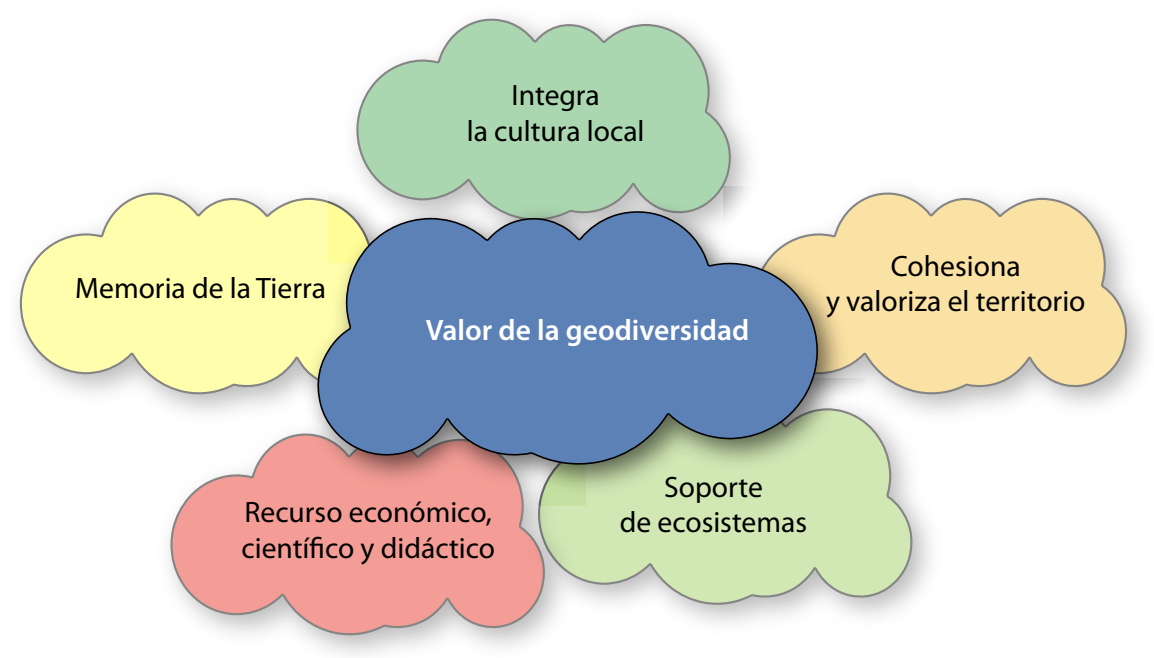

Nota. Elaboración personal con base en Educación ambiental y conservación de la geodiversidad. Junta de Andalucía, 2015, p. 15. 
y los cursos de postgrado, y realizarse a través de los planes de estudios escolares y la educación ambiental. También debería incluir la formación profesional y la orientación laboral. Pero también puede llegar a ser un asunto de cultura colectiva, a través de un incremento de la conciencia pública.

En este sentido, la incorporación del valor de la geodiversidad a través de la educación formal y no formal propiciaría un mayor reconocimiento y un acercamiento a la geología, geomorfología e hidrología local, con el fin de lograr que la comunidad entienda los vínculos existentes.

Para el mismo autor, las comunidades que busquen conservar y proteger su geodiversidad deberían iniciar:

[con] acciones encaminadas a cubrir las deficiencias en los planes de estudios de primaria y secundaria, en la educación ambiental, y en los programas de concienciación y sensibilización; en todos ellos debería considerarse adecuadamente un parámetro ambiental que aparece descuidado (el geológico), y tener en cuenta un nuevo concepto importante, la geoconservación, de acuerdo con los principios de la sostenibilidad.Estas acciones deberían situarse en una política enfocada a la renovación de los contenidos educativos y de los programas de formación. (TheodossiouDrandaki, 2000, p.121)

La inclusión de la educación ambiental en el sistema educativo, la cual incorpore la geodiversidad y la geoconservación entre las temáticas por tratar permitiría generar un cambio paulatino en la sociedad para lograr una vida más armónica y con un nivel de conflictos socioambientales más bajo. La principal meta de la educación ambiental, en relación con la geodiversidad, es dar a conocer a la ciudadanía la importancia del patrimonio geológico, para concientizarla sobre la necesidad de su conservación. No se debe olvidar que la geodiversidad, y en consecuencia el patrimonio geológico, implica, en definitiva, conservar la flora, la fauna $y$, en general, los ecosistemas que constituyen el espacio natural, logrando así una conservación integral del ambiente.

\section{Objetivo}

Considerando lo expresado, el objetivo del trabajo es presentar la educación ambiental como la estrategia principal para reducir los impactos producidos sobre la geodiversidad del sector serrano de Tandil.

\section{Metodología}

Con tal fin, se utilizó una metodología de tipo descriptiva y cualitativa, basada en la búsqueda bibliográfica; en primer lugar, para establecer los vínculos entre la educación ambiental y la geodiversidad;en segundo término, referidos a la situación en Tandil, respecto a la conservación de la geodiversidad.

\section{Desarrollo}

La ciudad de Tandil se encuentra a $380 \mathrm{Km}$ de la ciudad de Buenos Aires, capital de Argentina y está rodeada hacia el sur y el oeste por elevaciones cuyas alturas máximas apenas superan los $500 \mathrm{~m}$ s.n.m.

Cuenta con 124631 habitantes (último censo nacional realizado en el año 2010, INDEC) y en las últimas décadas ha tenido un importante crecimiento por disponer de servicios urbanos de importancia (educación, salud, etcétera), sumado a la tranquilidad de una localidad mediana y al paisaje serrano que propició el arribo de familias, principalmente desde el área metropolitana de Buenos Aires. 
Figura 2

Tandil, expansión urbana y zona serrana

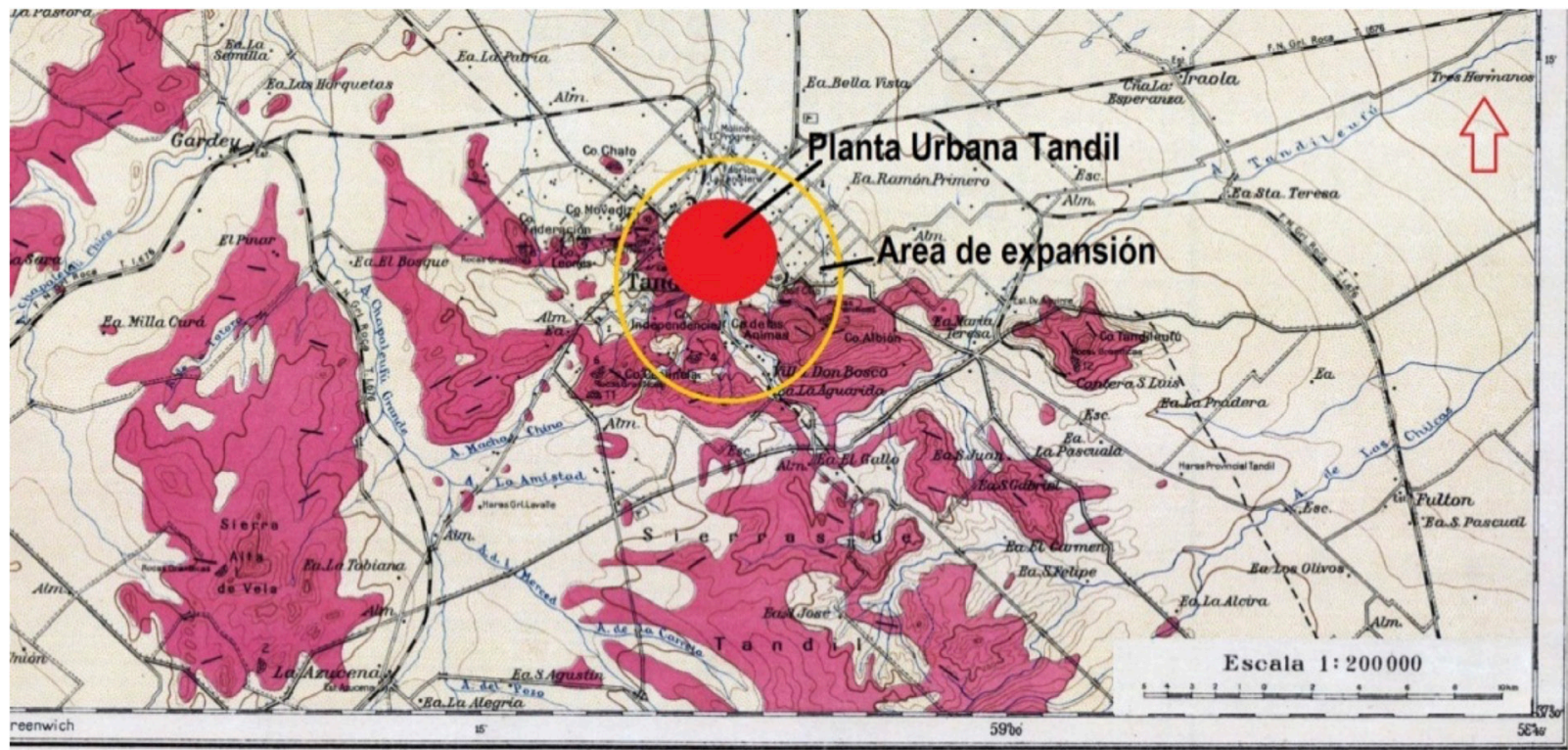

Notas. Adaptado deVillar Fabre, J.F., 1957. Descripción Geológica de la Hoja 32 q, Sierras del Tandil. Escala 1:200.000. Carta Geológico-Económica de la República Argentina. Provincia de Buenos Aires. Boletín 86, 46 p. Buenos Aires. Segemar (Servicio Geológico Minero Argentino).

En la figura 2, puede observarse como una ciudad compacta comenzó hace unos 20 años a expandirse hacia el sector norte, es decir hacia la llanura, pero también -producto de una valorización paisajística- hacia el sur y el oeste, y ocupa actualmente importantes sectores del área serrana. El sector inmobiliario y parte de la población igualó el poseer una mejor calidad de vida con residir en las sierras.

El avance urbano citado, propiciado por la expansión del uso urbano y turístico, produce modificaciones importantes del espacio serrano, las cuales implican problemáticas ambientales para el área de las sierras (es importante considerar que la zona no cuenta en general con servicios urbanos básicos, excepto electricidad) y el área circundante.

Es posible afirmar que las transformaciones producidas por el avance residencial se suman a los impactos generados por la actividad minera, que si bien fue suspendida dejo huellas significativas en el territorio (figura 3 ).

Además, las problemáticas en el área de las sierras se relacionan también con el vertido de efluentes cloacales, los cambios en la escorrentía superficial, tanto en cantidad de agua como en la dirección, cambios de las geoformas, impermeabilización del suelo por las construcciones y caminos. A lo anterior, se debe sumar el impacto sobre la biodiversidad existente.

Para el área de piedemonte (sector de depresiones, que se ha rellenado con sedimentos de los ríos) los cambios más importantes se asocian a la potencialidad de inundaciones producto de una reducción de las posibilidades de retención de las crecientes de los arroyos; esto ha obligado a la construcción 


\section{Figura 3}

\section{Construcciones y minería abandonada}

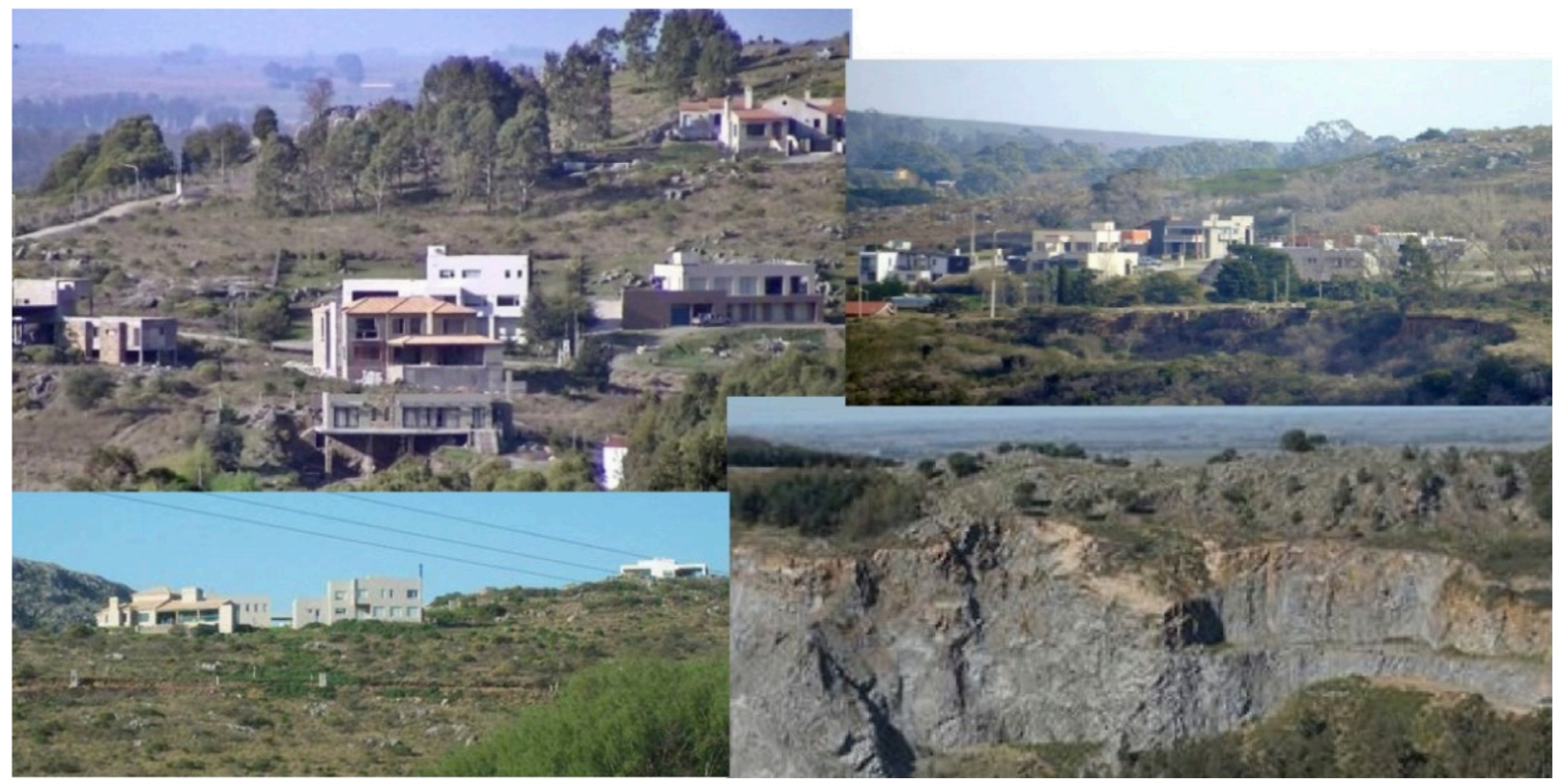

Nota. Fotografías de Aldo Ramos, septiembre 2020.

de diques secos para evitar inundaciones fuertes en el casco urbano.

Frente a las problemáticas señaladas, y producto del accionar de las ONG como la Asamblea Ciudadana en Defensa de las Sierras de Tandil, se generaron estrategias en pro del ambiente. Para proteger el área serrana del Partido de Tandil se estableció la Zona Protegida Natural Sierras de Tandil (ZPNST), pero las construcciones y el avance del turismo continúan a causa de los intereses de distintos sectores sociales (inmobiliarias, inversores, ciudadanos atraídos por el paisaje, antiguos dueños que pretenden mejorar su situación económica vendiendo terrenos comprados hace décadas a muy bajo precio a valores elevados, políticos que consideran que el crecimiento urbano es necesario, entre otros). Incluso en el Plan de Desarrollo Territorial (PDT) fue explicitada la necesidad de detener el avance sobre las sierras, pero eso no fue plasmado en el territorio.

Lo expresado de forma sintética indica que, pese a ciertas estrategias político-legales establecidas, no se ha logrado reducir la transformación de la geodiversidad de Tandil, que produce en definitiva impactos socioambientales negativos.

Por tanto, es necesario establecer un plan de acción desde la educación ambiental que, en primer lugar, permita dar a conocer el patrimonio geológico y geomorfológico del sistema serrano y los vínculos con la sociedad, para posteriormente valorizarlo y protegerlo.

De forma general, se menciona cómo es posible incorporar la temática de la geodiversidad en los tres tipos de educación, y específicamente profundizar en una propuesta para la educación formal. 
En primer lugar, es necesario incorporar el valor de la geodiversidad local en la educación formal. En el nivel preescolar (tres años de duración), primario (seis años) y secundario (seis años), se deben incorporar en el currículo contenidos relacionados al patrimonio geológico y geomorfológico, desde la perspectiva de la educación ambiental; es decir, desde un punto de vista reflexivo y crítico, no solo informativo.

Lo anterior, puede hacerse en las cátedras directamente (por ejemplo en orientaciones de Ciencias Naturales existe la materia Ciencias de la Tierra), pero también y fundamentalmente trabajando a través de proyectos que involucren distintas áreas de estudio como Química, Biología, Geografía, incluso Lengua y Literatura puede incluirse, ya que la geodiversidad está reflejada en la toponimia, las leyendas y los cuentos locales.

El nivel terciario y el universitario también deberían ser incluidos, aunque en este caso es más complejo ya que se trata de formaciones más específicas y no generalistas. No obstante, se puede incorporar con más fuerza el concepto de geodiversidad y geoconservación, al menos en las carreras afines: Geografía, Gestión Ambiental, Turismo, Paisajismo, entre otras, mediante el desarrollo de cursos o seminarios que puedan acreditarse como parte de las carreras. Incluso en este aspecto es posible establecer articulaciones entre la universidad y los institutos de educación terciaria. En este sentido, se han realizado desde la universidad algunos proyectos bajo el esquema de voluntariado, dentro del programa de la Secretaría de Políticas Universitarias, pero se enfocaron más sobre la biodiversidad. Se deberían fomentar estas acciones, pero acentuando en el valor de la geodiversidad y en la importancia de la geoconservación.

En segundo término, es necesario apuntar a la educación ambiental no formal a través de cursos, charlas, conferencias, etcétera, con el fin de lograr progresivamente difundir el valor de la geodiversidad e incentivar el interés por la historia de la Tierra. Si bien es posible que la asistencia del público general sea baja en un comienzo, debe ser considerado como un proceso continuo y permanente, por lo cual a mediano y largo plazo se podrá ver una mayor participación social.

Finalmente, también desde la educación ambiental informal, en la ciudad de Tandil, la Asamblea por la Preservación de las Sierras de Tandil se expresa de forma continua en los medios periodísticos locales (diarios, radios y canal), lo cual ha permitido así que el resto de la población conozca y reflexione sobre las problemáticas locales.

Por otra parte, la declaración del 2 de octubre como el Día de la Preservación de las Sierras (Ordenanza 12445/2011) colabora en la concientización de la importancia de la geodiversidad y la necesidad de su preservación. La Asamblea conmemora ese día habitualmente con caminatas, pero sería importante que el Municipio, las ONG e instituciones de distinto tipo generaran acciones para reforzar las actividades desarrolladas. Es posible, también el desarrollo de concursos fotográficos y la generación de espacios dotados de equipamiento de interpretación (geocircuitos urbanos, periurbanos y rurales) para promover una estrategia de comunicación que facilite al ciudadano el reconocimiento de la geodiversidad. Asociado a las iniciativas expuestas, sería importante desarrollar materiales para 
la divulgación y difusión de la geodiversidad y su importancia como parte del patrimonio natural, lo cual actualmente es escaso. Así como considerar que en las actividades de recreación (senderismo, cabalgatas, escala$\mathrm{da}$, entre otras), tanto para visitantes como para la población local, se incluyan acciones que valoricen la geodiversidad.

\section{Propuesta de educación ambiental para la geodiversidad en el nivel secundario superior}

La educación secundaria en Argentina se divide en secundaria básica (primero, segundo y tercer año) y secundaria superior (cuarto, quinto y sexto año, sétimo en escuelas técnicas).

La propuesta presentada puede adaptarse a cualquiera de los tres años de la secundaria superior. Si bien la educación ambiental debe ser un proceso continuo que en el ámbito de la educación formal debe iniciar desde el preescolar y continuar hasta que finalice la educación obligatoria (17/18 años de edad), en este caso específico se presenta una propuesta para un año de la educación formal, pero la misma debe estar integrada a un proyecto general del distrito educativo para que el alumno pueda seguirlo durante su trayectoria educativa, independientemente de la escuela a la que concurra. El tema del proyecto es Concientizando sobre el valor de la geodiversidad, su fundamentación se enmarca en la necesidad de que la sociedad incorpore la geodiversidad del sistema serrano como un elemento de valor, más allá de lo paisajístico, para que se permita realmente un accionar responsable hacia el ambiente. La meta es formar ciudadanos que reflexionen en su accionar presente y futuro.
Los objetivos del proyecto son: a. reconocer el valor de la geodiversidad, b. entender los vínculos de la geodiversidad con la biodiversidad y con el hombre, los cuales apuntan a un reconocimiento del ambiente que nos rodea y al mismo tiempo entender las interacciones que se producen en el mismo.

Como lugar para el desarrollo de las actividades se utiliza la Reserva Natural Sierra del Tigre, 142 hectáreas dentro del área de estudio mencionada en la figura 2. Es un área de fácil acceso para que puedan concurrir los alumnos de establecimientos educativos.

La propuesta consiste en el abordaje de los objetivos propuestos desde diferentes materias curriculares. En este caso se presentan algunas, no es necesario que todas participen; no obstante, es posible integrarlas a todas, al menos en alguno de los contenidos abordados durante el año.

La propuesta debe implicar dos fases necesariamente. Una abordada en el aula y la otra que implique al menos una salida de campo en el año que permita observar y aplicar aquellos contenidos trabajados en la escuela.

Para evaluar los aportes de cada materia se considera la tabla 1, donde se presentan los elementos que integran la geodiversidad.

El trabajo realizado desde diferentes perspectivas debe ser integrado en un taller final cuyo objetivo sea la construcción de un pensamiento crítico que tienda a la formación de prácticas, valores y actitudes que, individual y colectivamente, aporten a la identificación y la comprensión de los problemas asociados a la geodiversidad. 


\section{Tabla 1}

Elementos integradores de la geodiversidad

\begin{tabular}{|c|c|}
\hline Materia & Contenidos a abordar \\
\hline \multirow{3}{*}{ Geografía } & Breve información de la geología local. \\
\hline & $\begin{array}{l}\text { Relaciones de la geodiversidad con las activi- } \\
\text { dades económicas (por ejemplo minería). }\end{array}$ \\
\hline & $\begin{array}{l}\text { Expansión urbana sobre el área serrana, anali- } \\
\text { zando la infraestructura urbana necesaria. }\end{array}$ \\
\hline Historia & $\begin{array}{l}\text { Relación de la geodiversidad con el trabajo } \\
\text { picapedrero de fines del siglo XIX y principios } \\
\text { del XX. }\end{array}$ \\
\hline \multirow[t]{2}{*}{ Biología } & $\begin{array}{l}\text { Análisis de los vínculos establecidos entre los } \\
\text { ecosistemas y la geodiversidad (por ejemplo } \\
\text { el reciente descubrimiento de una lagartija - } \\
\text { Liolaemus tandiliensis- que tiene hábitos saxíco- } \\
\text { las, es decir vive en ambientes rocosos. }\end{array}$ \\
\hline & $\begin{array}{l}\text { Análisis del impacto sobre las estructuras roco- } \\
\text { sas de especies exóticas. }\end{array}$ \\
\hline Literatura & $\begin{array}{l}\text { Análisis de leyendas y mitos en donde la geodi- } \\
\text { versidad está presente. Por ejemplo en la zona } \\
\text { existen rocas que producto de la exfoliación } \\
\text { esferoidal quedan erguidas y han sido com- } \\
\text { paradas con centinelas, dando lugar a escritos. }\end{array}$ \\
\hline $\begin{array}{l}\text { Introducción } \\
\text { a la física }\end{array}$ & $\begin{array}{l}\text { Análisis de fenómenos como gravedad asocia- } \\
\text { do a los movimientos de rocas. }\end{array}$ \\
\hline Matemática & $\begin{array}{l}\text { Cálculo y evaluación de superficies transforma- } \\
\text { das por el hombre en el espacio serrano. }\end{array}$ \\
\hline $\begin{array}{l}\text { Educación } \\
\text { física }\end{array}$ & $\begin{array}{l}\text { Realización y/o colaboración de las actividades } \\
\text { de campo. }\end{array}$ \\
\hline
\end{tabular}

Nota. Elaboración propia.

En esta instancia, que puede contar con varios encuentros hacia el final del año, el alumno discute los diferentes valores que presenta la geodiversidad y la necesidad de conservación, para alcanzar así un reconocimiento de las múltiples y complejas relaciones ecológicas, políticas, culturales y económicas que integran la problemática.

\section{Reflexiones finales}

A partir de lo expresado, se subraya la importancia de la geodiversidad al representar la memoria de la Tierra y posibilitar la cohesión de un territorio, por tanto es fundamental su conservación. No obstante, en muchas ocasiones, exceptuando las áreas protegidas, es difícil lograr el objetivo de su resguardo, por presiones de diferentes actores sociales.

Por tanto, se considera que la educación ambiental, desplegada en todos sus posibles niveles de acción, es la estrategia que verdaderamente puede llegar a producir el cambio necesario en la sociedad para alcanzar una concientización sobre su valor en Tandil en este caso específico y de manera general en cualquier sitio con una problemática similar, sin olvidar otras herramientas, pero considerando que el cambio de actitud y comportamiento de la comunidad es lo que permitirá una verdadera geoconservación.

\section{Referencias}

Carcavilla, L., Durán, J.J. y López-Martínez, J. (2008). Geodiversidad: concepto y relación con el patrimonio geológico. Geo-Temas, 10, 1299-1303. Las Palmas de Gran Canaria: VII Congreso Geológico de España.

Nieto, L. M. (2001). Geodiversidad: propuesta de una definición integradora. Boletín Geológico y Minero, Vol. 112, Núm. 2, pp. 3-12.

Serrano Cañadas, E. y Ruiz Flaño, P. (2007). Geodiversidad: concepto, evaluación y aplicación territorial. El caso de Tiermes Caracena (Soria). Boletín de la A.G.E. N. 45 . pp. 79-98.

Theodossiou-Drandaki, I. (2000). Sin educación no es posible la conservación. En Patrimonio Geológico: Conservación y Gestión. D. Barettino, W. A. P. Wimbledon y E. Gallego (eds.), pp. 119-135. Madrid.

Voth, A. (2008). Los geoparques y el geoturismo: nuevos conceptos de valorización de recursos patrimoniales y desarrollo regional. XI Coloquio Ibérico de Geografía. Alcalá de Henares. 